INPLASY

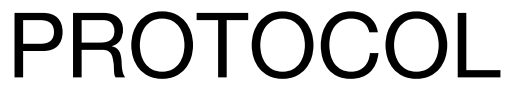

To cite: Liu et al. Comparison of robot-assisted surgery, laparoscopic-assisted surgery, and conventional open surgery for the treatment of gastric cancer: A network metaanalysis. Inplasy protocol 2021120121. doi:

10.37766/inplasy2021.12.0121

Received: 27 December 2021

Published: 27 December 2021

Corresponding author:

Liu Y

642508200@qq.com

Author Affiliation:

Department of General

Surgery, The Affiliated Wuxi

People's Hospital of Nanjing

Medical University, Wuxi,

China.

Support: Wei Shen.

Review Stage at time of this submission: Preliminary

searches.

Conflicts of interest:

None declared.

\section{Comparison of robot-assisted surgery, laparoscopic-assisted surgery, and conventional open surgery for the treatment of gastric cancer: A network meta-analysis}

Liu, Y1; Tian, ZQ2; Song, C3; Zhang, YC4; Shen, W5.

Review question / Objective: Gastric cancer is a highly prevalent malignancy, mainly in China, Korea, and Japan. Over the past few decades, significant advances have been made in surgical techniques to treat gastric cancer. The transition from conventional open distal gastrectomy to laparoscopic procedures has evolved to robotic-assisted treatment. However, the effectiveness of robotic-assisted gastric cancer treatment is not clear. This study demonstrates the effectiveness of robotic-assisted treatment by comparing it with laparoscopic-assisted treatment and open radical gastric cancer surgery. p: patients with gastric cancer, I: roboticassisted treatment, $\mathrm{C}$ : open radical gastric cancer surgery, laparoscopic radical gastric cancer surgery, 0 : postoperative complication incidence and operative time, length of hospital stay, etc., S. Randomised controlled trials or cohort studies.

INPLASY registration number: This protocol was registered with the International Platform of Registered Systematic Review and Meta-Analysis Protocols (INPLASY) on 27 December 2021 and was last updated on 27 December 2021 (registration number INPLASY2021120121).

\section{INTRODUCTION}

Review question / Objective: Gastric cancer is a highly prevalent malignancy, mainly in China, Korea, and Japan. Over the past few decades, significant advances have been made in surgical techniques to treat gastric cancer. The transition from conventional open distal gastrectomy to laparoscopic procedures has evolved to robotic-assisted treatment. However, the effectiveness of robotic-assisted gastric cancer treatment is not clear. This study demonstrates the effectiveness of robotic- 
assisted treatment by comparing it with laparoscopic-assisted treatment and open radical gastric cancer surgery. $p$ : patients with gastric cancer, I: robotic-assisted treatment, C: open radical gastric cancer surgery, laparoscopic radical gastric cancer surgery, 0 : postoperative complication incidence and operative time, length of hospital stay, etc., S. Randomised controlled trials or cohort studies.

Condition being studied: The incidence of gastric cancer is increasing every year and the only treatment available is surgical intervention, thus requiring continuous development of surgical techniques. The advent of robotic technology is a breakthrough in minimally invasive surgical techniques, but patient prognosis is not clear, and there is a lack of large sample, multicentre studies to confirm the efficiency of robotic-assisted treatment techniques in the treatment of gastric cancer. Therefore, this study demonstrates the feasibility and safety of robotic distal gastrectomy for gastric cancer by comparing multiple surgical approaches.

\section{METHODS}

Search strategy: We searched all Englishlanguage literature from PubMed, Embase, Cochrane Library, Ovid, and other literature databases for transcatheter, laparoscopicassisted treatment, and da Vinci robotassisted D2 distal gastrectomy for gastric cancer. At the same time, the references included in the literature were searched to supplement the relevant literature. English search terms had Robot gastrectomy, Da Vinci Robot, laparoscopy, laparoscopic, stomach neoplasms, gastric cancer, gastric carcinoma, and stomach cancer. The search period is from 2015 to December 2021. Inclusion criteria Study type: RCT or non-RCT-based literature, language limited to English. Subjects: Patients with a postoperative pathological or cytological confirmation of gastric cancer. Outcome indicators: The direct observation was patients' postoperative symptoms and complications (including postoperative pneumonia, anastomotic leakage, and ileus, etc.), and the second observation was patients' operative time and length of hospital stay. Control measures: Three surgical procedures included conventional open distal gastrectomy, laparoscopicassisted distal gastrectomy, and robotassisted distal gastrectomy. Exclusion criteria Type of study: Literature that does not account for the kind of study or where the type of study does not match. Type of literature: literature that does not match the kind of literature, such as reviews, meta-analyses, non-comparative studies, conference reports, etc. Outcome indicators: literature from which valid outcome data could not be extracted and literature in which the hands studied in the literature did not match. Control measures: literature with only a single surgical procedure or no surgical procedure for comparison. Subjects: literature that included patients with comorbid other malignancies or who could not tolerate surgery. Additional screening criteria: literature with too small a sample size or poor experimental design.

Participant or population: Stomach cancer patients.

Intervention: Robot-assisted distal gastrectomy.

Comparator: Conventional open distal gastrectomy, laparoscopic-assisted distal gastrectomy.

Study designs to be included: Randomised controlled trials or cohort studies.

Eligibility criteria: Inclusion criteriaStudy type: RCT or non-RCT-based literature, language limited to English. Subjects: Patients with a postoperative pathological or cytological confirmation of gastric cancer. Outcome indicators: The direct observation was patients' postoperative symptoms and complications (including postoperative pneumonia, anastomotic leakage, and ileus, etc.), and the second observation was patients' operative time and length of hospital stay. Control measures: Three surgical procedures included conventional open distal gastrectomy, laparoscopic-assisted distal 
gastrectomy, and robot-assisted distal gastrectomy. Exclusion criteriaType of study: Literature that does not account for the kind of study or where the type of study does not match. Type of literature: literature that does not match the kind of literature, such as reviews, meta-analyses, non-comparative studies, conference reports, etc. Outcome indicators: literature from which valid outcome data could not be extracted and literature in which the hands studied in the literature did not match. Control measures: literature with only a single surgical procedure or no surgical procedure for comparison. Subjects: literature that included patients with comorbid other malignancies or who could not tolerate surgery. Additional screening criteria: literature with too small a sample size or poor experimental design.

Information sources: PubMed, Embase, Cochrane Library, Ovid, and other literature databases.

Main outcome(s): Operative time, postoperative hospital stay and Incidence of postoperative complications.

Quality assessment / Risk of bias analysis: The included literature will be evaluated for methodological quality according to the NOS scale and the JADAD scale.

Strategy of data synthesis: Direct metaanalysis was performed using Review Manager 5.4 software to draw forest plots, with effect sizes odds ratio (OR) and $95 \%$ confidence interval (CI) expressed for dichotomous variable information; and mean difference (MD) and $95 \%$ confidence interval (Cl) expressed for continuous varying effect sizes. A network metaanalysis was performed using R-Studio software (JAGS 4.3.0). The net relationship between the interventions was plotted. $A X^{2}$ test and $I^{2}$ quantification were performed to determine the magnitude of heterogeneity. When $\mathrm{I}^{2} \leq \mathbf{5 0} \%$, heterogeneity was ignored; $I^{2}>50 \%-70 \%$, there was moderate heterogeneity; $I^{2}>70 \%$ was high heterogeneity, and a random-effects model was required. Draw forest plots and ranked probability plots to compare the advantages and disadvantages of various surgical approaches.

Subgroup analysis: Patients are divided into subgroups according to their country, gender, age, economic status and family factors.

Country(ies) involved: China.

Keywords: gastric cancer; laparoscopically assisted gastrectomy; robot-assisted gastrectomy.

Contributions of each author:

Author 1 - Liu Y.

Author 2 - Tian ZQ.

Author 3 - Song C.

Author 4 - Zhang YC.

Author 5 - Shen W. 